\title{
Sensing with TRP channels
}

\author{
Thomas Voets, Karel Talavera, Grzegorz Owsianik \& Bernd Nilius
}

\begin{abstract}
Drosophila melanogaster [AU: OK to list full species name - this is as meant?] flies carrying the trp (transient receptor potential) mutation are rapidly blinded by bright light, because of the absence of a $\mathrm{Ca}^{2+}$-permeable ion channel in their photoreceptors. The identification of the trp gene and the search for homologs in yeast, flies, worms, zebrafish and mammals has led to the discovery of a large superfamily of related cation channels, named TRP channels. Activation of TRP channels is highly sensitive to a variety of chemical and physical stimuli, allowing them to function as dedicated biological sensors that are essential in processes such as vision, taste, tactile sensation and hearing.
\end{abstract}

All living cells are surrounded by a cell membrane, an oily double layer of phospholipids that holds the essential cellular components together ${ }^{1}$. Because of its hydrophobic nature, a pure phospholipid bilayer is virtually impermeable to small charged molecules and ions such as $\mathrm{Na}^{+}$, $\mathrm{K}^{+}, \mathrm{Ca}^{2+}$ or $\mathrm{Cl}^{-}$. Yet, for their proper functioning, cellular membranes must regulate passage of these ions. To achieve this, cellular membranes contain various ion channels, which are proteinaceous pores that allow rapid permeation of ions across the phospholipid bilayer in a highly regulated manner ${ }^{1,2}$. The flow of ions through ion channels can evoke swift electrical signals and provoke rapid changes in the concentration of second messengers such as $\mathrm{Ca}^{2+}$. As such, ion channels form the basis of many crucial biological processes, including the beating of the heart and the rapid signaling in nerve cells ${ }^{1}$.

The human genome encodes hundreds of ion channels, which can be subdivided into a few dozen channel families with strongly divergent structures and functional features ${ }^{1}$. The functioning of an ion channel is mainly determined by two key characteristics: the type of ions that can permeate the pore ('channel selectivity') and the signals that regulate opening and closing of the pore ('channel gating'). In this review, we describe the TRP superfamily, a specific class of ion channels that gate in response to a diverse array [AU: OK as edited?] of chemical and physical stimuli. Because of this gating promiscuity, TRP channels serve as versatile sensors that allow individual cells and entire organisms to detect changes in their environment. After a brief overview of the TRP superfamily, we focus on the role of TRP channels as thermo-, chemoand mechanosensors.

\section{A short trip through the TRP superfamily}

TRP history began in 1969, when Cosens and Manning discovered a Drosophila mutant that showed a transient instead of a sustained response to bright light ${ }^{3}$. Analysis of the photoreceptor cells of mutant flies revealed that sustained exposure to light induced a brief rather than the normal plateau-like receptor potential.[AU: OK as edited for clarity, flow, and to avoid word repetition?] The mutant was thus bap-

Laboratory of Physiology, Campus Gasthuisberg O\&N, KU Leuven, B-3000 Leuven, Belgium. Correspondence should be addressed to T.V. (Thomas.voets@med.kuleuven.ac.be). tized $\operatorname{trp}$, for transient receptor potential. Two decades later, the trp gene was cloned ${ }^{4}$ and later shown to encode $\mathrm{Ca}^{2+}$-permeable cation channel, $\mathrm{TRP}^{5}$.[AU: Again, $\mathrm{OK}$ as edited to tighten? It seems clear already that TRP was the first member of the TRP family from the history described.] Subsequently, two close TRP homologs, named TRPL (or TRP-like $)^{6}$ and TRP $\gamma$ (ref. 7), were identified in Drosophila, and all three proteins were found to contribute to the light-induced currents in the photoreceptor cells ${ }^{6-8}$. Drosophila TRP functions as a receptor-operated channel that is activated downstream of the light-induced, phospholipase $\mathrm{C}$-mediated hydrolysis of phosphatidylinositol 4,5-bisphosphate $\left(\mathrm{PIP}_{2}\right.$; ref. 9). It is still unknown whether TRP opens in response to reduced $\mathrm{PIP}_{2}$ levels, or whether it is activated by diacyl glycerol (DAG) or by polyunsaturated fatty acids derived from $\mathrm{DAG}^{10}$.

The identification of the first mammalian TRP homologs in 1995 fueled the quest for trp-related genes, which resulted in the identification and characterization of more than 50 TRP channels in yeast, worms, insects, fish and mammals ${ }^{11,12}$. Thanks to these genome sequencing efforts, we now know that there are 28 trp-related genes in mice, 27 in humans, 17 in the worm Caenorhabiditis elegans and 13 in Drosophila.[AU: Previous two sentences $\mathrm{OK}$ as edited to tighten and avoid word repetition?] TRP channels can be classified into seven subfamilies: TRPC, TRPV, TRPM, TRPA, TRPP, TRPML and TRPN (Fig. 1a). The TRPC subfamily ('C' stands for canonical or classical) contains proteins with the highest homology to the Drosophila TRP protein[AU: $\mathrm{OK}$ as edited? (Proteins would be homologous to the TRP protein, not gene, correct?)]. The other subfamilies were named after their first identified members: the TRPV subfamily after the vanilloid receptor 1 (VR1, now TRPV1), the TRPM subfamily after the tumor suppressor melastatin (TRPM1), the TRPA subfamily after the protein denoted ankyrin-like with transmembrane domains 1 (ANKTM1, now TRPA1), TRPN after the no mechanoreceptor potential C (nompC) gene from Drosophila, TRPP after the polycystic kidney disease-related protein 2 (PKD2, now TRPP2), and TRPML after mucolipin 1 (TRPML1). For a detailed overview of the members of the different subfamilies and their putative roles, we refer to other recent reviews ${ }^{13-15}$

The basic architecture of TRP channels is the same as that of voltagegated $\mathrm{K}^{+}$channels ${ }^{16}$ : four identical or similar subunits with six transmembrane domains (TM1-TM6) and cytosolic N- and C-terminal tails tetramerize to form a functional channel ${ }^{17}$. TM5, TM6 and the con- 
a

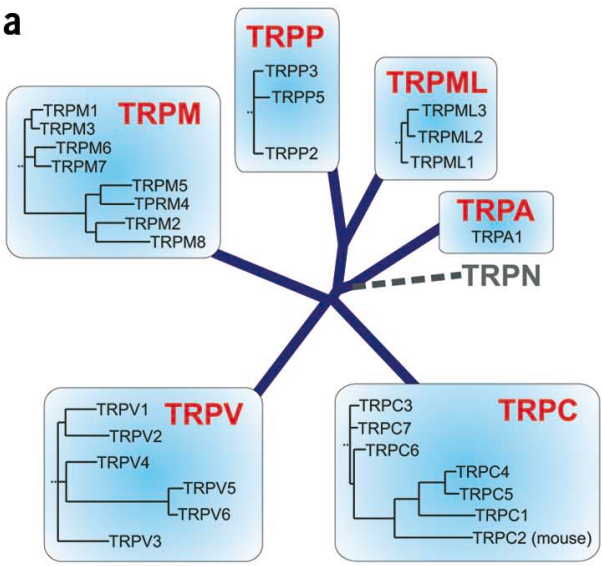

b

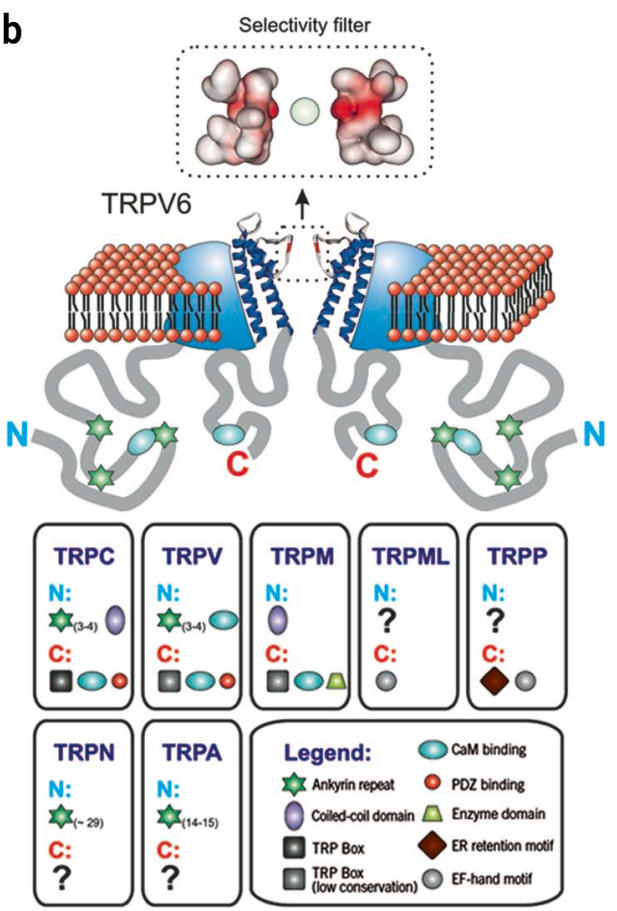

Figure 1 Phylogeny and architecture of TRP channels. (a) Phylogenetic relationships between members of the human TRP-channel superfamily. The multiple-alignment phylogenetic tree illustrates the relation between the different TRP subfamilies. Phenograms were generated independently for each subfamily. Note that TRPC2 is a pseudogene in primates and that TRPN channels have not been identified in mammals. (b) Top, proposed architecture of TRPV6. The pore region is formed by the loop between TM5 and TM6, which forms the pore helix and selectivity filter ${ }^{18}$ similar to that of $\mathrm{K}^{+}$channels $^{16}$. The structures of TM1-TM4 are currently unknown. Four identical or similar subunits form a functional channel (for clarity, only two subunits are shown). Bottom, overview of structural motifs found in the $\mathrm{N}$ and $\mathrm{C}$-terminal tails of the different TRP subfamilies. The number of motif repeats is given in brackets. The structural motifs in the cytosolic tails of members of the TRPML, TRPP, TRPN and TRPA subfamilies are not always known, hence the question marks.

necting pore loop form the central cation-conducting pore ${ }^{18}$, whereas TM1-TM4 and the cytoplasmic N- and C-terminal parts are thought to contain the regulatory domains that control channel gating (Fig. 1b). Compared with the six TM channels, TRP channels can have extremely long cytoplasmic $\mathrm{N}$ - and $\mathrm{C}$-terminal tails containing several regulatory

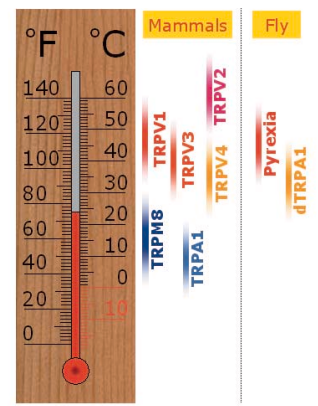

Figure 2 Activation range of human and Drosophila thermoTRPs. Indicative temperature range for activation of mammalian and Drosophila thermoTRPs in heterologous expression systems. Note that TRPM8 and TRPA1 are activatedupon cooling, whereas all other indicated channels are heat activated.

modules ${ }^{14,15}$ and, as in TRPM2, TRPM6 and TRPM7, can even contain entire functional enzymes ${ }^{19}$. The relevance of these different cytoplasmic domains to channel functioning is poorly understood.

\section{TRP channels as thermosensors}

Partially on the basis of the functional properties of Drosophila TRP, mammalian TRP homologs were initially solely envisaged as the molecular correlates of PLC-dependent or store-operated cation channels ${ }^{11,12,20-23}$.[AU: Sentence OK as edited?] This view changed when Caterina and colleagues used an expression-cloning strategy to search for a receptor for capsaicin, the pungent substance in hot chili peppers. They isolated a cDNA clone from sensory neurons that encodes VR1 (capsaicin is a vanilloid compound), $\mathrm{C} \mathrm{Ca}^{2+}$-permeable cation channel that could be activated not only by capsaicin and but also by noxious heat $\left(>43^{\circ} \mathrm{C}\right.$; ref. 24$)$. Surprisingly, VR1 did not show homology to known ligand-gated channels, but appeared to be most closely related to TRP. Indeed, the renaming of VR1 in the unified TRP nomenclature as TRPV1 reflects its status as the first recognized member of the TRPV subfamily ${ }^{25}$.[AU: Sentence OK as edited for flow?]

Six mammalian thermoTRPs. Since the discovery of TRPV1 as a heatactivated channel, five additional mammalian temperature-sensitive TRP channels (or thermoTRPs ${ }^{26}$ ) have been described. TRPV1 and its closest homologs, TRPV2 (ref. 27), TRPV3 (refs. 28-30) and TRPV4 (refs. 31,32), are activated upon heating, whereas TRPM8 (refs. 33,34) and TRPA1 (ref. 35), two more distantly related TRP channels belonging to distinct subfamilies, are activated upon cooling. (However, it should be noted that the cold activation of TRPA1 has been questioned ${ }^{36}$.) Together, these thermoTRPs have the potential to detect changes in temperature from $<10$ to $>50{ }^{\circ} \mathrm{C}$ (Fig. 2), which corresponds to the physiological range of temperatures that humans can discriminate. The indicated range for thermal activation of the different thermoTRPs should not be taken too strictly, however, as the sensitivity for thermal activation of these channels can be substantially modified by cellular and environmental factors. For example, a reduction of cellular $\mathrm{PIP}_{2}$ levels, such as occurs upon activation of the $\mathrm{G}$ protein-coupled receptors that activate phospholipase $\mathrm{C}$, increases the heat sensitivity of TRPV1 (refs. 37,38) while also causing desensitization of the coldactivated TRPM8 (refs. 39,40).

Mechanisms of thermosensation. It is important to briefly consider how specialized the temperature sensitivity of thermoTRPs actually is.[AU: OK as edited for conciseness?] In general, the temperature 
a

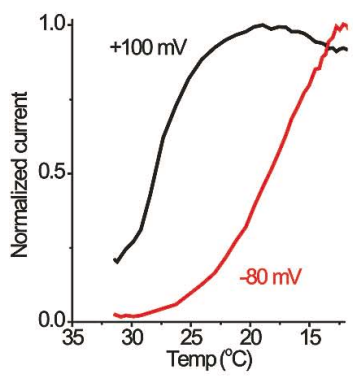

b

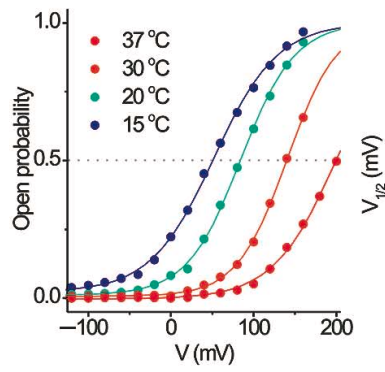

C

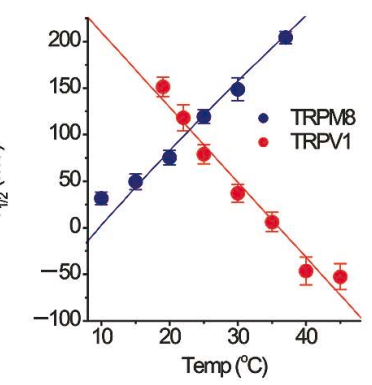

Figure 3 Temperature sensitivity is voltage dependent. (a) Normalized TRPM8 current in response to cooling at +100 and $-80 \mathrm{mV}$. Note that at depolarized potentials, current activation occurs at higher temperatures than at more negative potentials. (b) Plot showing the open probability of TRPM8 in function of voltage at the indicated temperatures. (c) Plot of the midpoint of the activation curves $\left(V_{1 / 2}\right)$ versus temperature for TRPV1 and TRPM8. Adapted from ref. 41.

dependence of a reaction rate can be quantified with the 10-degree temperature coefficient, or $Q_{10}$, which is defined as $Q_{10}=\operatorname{rate}(T+10) /$ $\operatorname{rate}(T)$ (ref. 1).[AU: Correct as edited? We do not use superscripted reference citations next to numbers or variables where they might be confusing.] All ion channels, like all other types of enzymes, show some degree of temperature dependence. The ionic flux through an open channel increases with temperature, with typical $Q_{10}$ values ranging between 1.2 and 1.4, which can be accounted for by the temperature dependence of ionic diffusion ${ }^{1}$. Voltage-dependent gating of classical voltage-gated channels shows $Q_{10}$ values that are typically between 2 and 4 (ref. 1). However, quantification of the temperature dependence of the ion flux through heat-activated thermoTRPs has yielded $Q_{10}$ values between 6 and 30 (refs. 24,27-30,32). Moreover, in the coldactivated thermoTRPs, TRPM8 and TRPA1, ionic currents decrease when temperature increases ${ }^{33-35}$, a fact that, in theory, corresponds to $Q_{10}$ values $<1$. Clearly, thermoTRPs must be specialized to detect and discriminate small deviations in temperature.

Several possible mechanisms could explain the remarkable temperature sensitivity of thermoTRPs ${ }^{15}$. First, changes in temperature could lead to the production of channel-activating ligands. In such a model, the ligand-producing enzyme rather than the thermoTRP itself would be temperature dependent. Given that the thermal sensitivity of most thermoTRPs is well preserved in cell-free membranes, temperaturedependent ligand binding is unlikely to be a general mechanism for thermosensation in TRPs. However, in the case of TRPV4, heat activation no longer occurs in cell-free inside-out patches, suggesting that some crucial soluble messenger is lost in the cell-free system ${ }^{32}$ [AU: Sentence $\mathrm{OK}$ as edited?]. Second, channel activation could result from a temperature-dependent phase transition of the lipid membrane or a conformational transition (or denaturation) of the channel protein structure. Phase transitions of the lipid membrane or conformational transitions in proteins usually occur over a narrow temperature range, which could potentially explain the steep temperature dependence of thermoTRP activation. At present, however, experimental evidence in support of such a mechanism does not exist.

A recent study presented a fundamental thermodynamic principle to explain cold activation of TRPM8 and heat activation of TRPV1, which does not necessitate diffusible messengers or conformational transitions $^{41}$. It was found that the temperature sensitivity of these channels is strongly dependent on the transmembrane voltage. At depolarized potentials, TRPM8 is activated at much higher temperatures than at more physiological, negative potentials (Fig. 3a; refs. 41,42). Similarly,
TRPV1 is activated at much lower temperatures when the membrane is depolarized than when it is hyperpolarized. Further analysis revealed that TRPM8 and TRPV1 are voltagegated channels activated upon membrane depolarization (Fig. 3b). Thermal activation reflects a robust but graded shift of the voltage dependence of activation from strongly depolarized potentials toward the physiological potential range (Fig. 3b,c). This finding has several important implications. First, it implies that a thermal threshold is not the optimal parameter to describe thermoTRPs: the thermal sensitivity of these channels depends on voltage, and temperature-dependent activation represents a gradual increase in the probability of a channel being open [AU: $\mathrm{OK}$ as edited?] rather than a threshold phenomenon. Second, it strongly argues against temperature-dependent phase transition of the lipid membrane or conformational transitions of the channel protein as mechanisms for thermal activation, as such processes would predict a single sharp thermal threshold ${ }^{41}$.

A relatively[AU: $\mathrm{OK}$ as edited to avoid repetition of 'surprisingly, used earlier?] simple two-state model was found to accurately reproduce the temperature-dependent activation of TRPV1 and TRPM8 (Box 1; ref. 41). For TRPM8, the temperature dependence of channel opening $\left(Q_{10} \sim 1.2\right)$ is much less steep than that of channel closing $\left(Q_{10}\right.$ $\sim 9.4$ ), which leads to channel activation upon cooling. In the case of TRPV1, channel opening shows a much steeper temperature dependence $\left(Q_{10} \sim 14.8\right)$ than channel closing $\left(Q_{10} \sim 1.35\right)$, leading to channel activation upon heating ${ }^{41}$. A detailed thermodynamic analysis (Box 1) reveals that channel opening is associated with a decrease in entropy in cold-activated channels, and with an increase in entropy in heat-activated channels. An interesting and testable outcome of this theoretical analysis is that the strong temperature dependence of thermoTRPs is correlated with the low gating charge of their voltage sensor.

Life without thermometers. Important new insight into the physiology of thermosensation has been obtained from the study of genetically modified mice that lack expression of specific thermoTRPs. The classical view was that ambient temperature is sensed by neurons from the dorsal root ganglia (DRG) that have thermosensitive projections in the $\operatorname{skin}^{26}$. Distinct subsets of DRG neurons express different thermoTRPs, endowing them with distinct thermosensitive properties. In line with this, DRGs from TRPV1-deficient mice specifically lack the subset of neurons that respond to moderate heat $\left(\sim 43^{\circ} \mathrm{C}\right)$, whereas responses to temperatures $>55{ }^{\circ} \mathrm{C}$ were preserved. Behaviorally, TRPV1-deficient mice have a significantly[AU: Present tense OK, for consistency throughout paragraph? Also, please confirm that this finding is in fact statistically significant.] delayed response to painful heat, for example upon tail immersion in hot water or when placed on a hot plate, but have normal responses to mechanical stimuli ${ }^{43}$. Additionally, TRPV1knockout mice do not develop increased sensitivity to heat in response to inflammation ${ }^{43,44}$. This confirms the hypothesis that inflammationinduced thermal hyperalgesia reflects the increased sensitivity of TRPV1 induced by constituents of the so-called 'inflammatory soup', such as protons, bradykinin, nerve growth factor and prostaglandins.

TRPV3, a thermoTRP activated by moderate heat, is strongly expressed in keratinocytes in the $\operatorname{skin}^{28,29}$, a tissue that was not considered to be involved in thermosensing. Yet, mice lacking TRPV3 have significant deficits in the sensing of warm temperatures and noxious 


\section{REVIEW}

\section{BOX 1: A SIMPLE MODEL FOR THERMOSENSITIVITY}

Assume the simplest kinetic scheme for a voltage-gated channel.

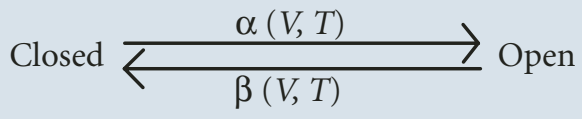

Following Eyring rate theory (see ref. 39 for an analogous analysis using Arrhenius activation energy), $\alpha$ and $\beta$ are related to membrane voltage and temperature according to

$$
\alpha=\kappa \frac{k T}{h} \cdot \mathrm{e}^{\frac{-\Delta H_{\text {open }}+T \Delta S_{\text {open }}+\delta z F V}{\mathrm{RT}}} \quad \beta=\kappa \frac{k T}{h} \cdot \mathrm{e}^{\frac{-\Delta H_{\text {close }}+T \Delta S_{\text {close }}-(1-\delta) z F V}{\mathrm{RT}}}
$$

where $\Delta H_{\text {open }}$ and $\Delta H_{\text {close }}$ represent the enthalpies and $\Delta S_{\text {open }}$ and $\Delta S_{\text {close }}$ the entropies associated with channel opening and closing, respectively. $R$ represents the gas constant $\left(8.31 \mathrm{~J} \mathrm{~mol}^{-1} \mathrm{~K}^{-1}\right), T$ the absolute temperature, $z$ the effective charge associated with voltagedependent gating, $\delta$ the fraction of $z$ moved in the outward direction, $F$ the Faraday constant $\left(9.65 \times 10^{4} \mathrm{C} \mathrm{mol}^{-1}\right), \mathrm{kBoltzmann} \mathrm{s}$ constant $\left(1.381 \times 10^{23} \mathrm{~J} \mathrm{~K}^{-1}\right)$ and $h$ Planck's constant $\left(6.626 \times 10^{-34} \mathrm{~J} \mathrm{~s}\right) . \kappa$ is the transmission coefficient, which is usually assumed to equal 1 .

[AU: Figure 5, the figure within Box 1, must be cited somewhere in the text of the box, so we have added a citation to the next sentence. Please confirm or suggest an alternative place to mention this. Also, please provide a title, and optionally a brief legend, for Fig. 5.]
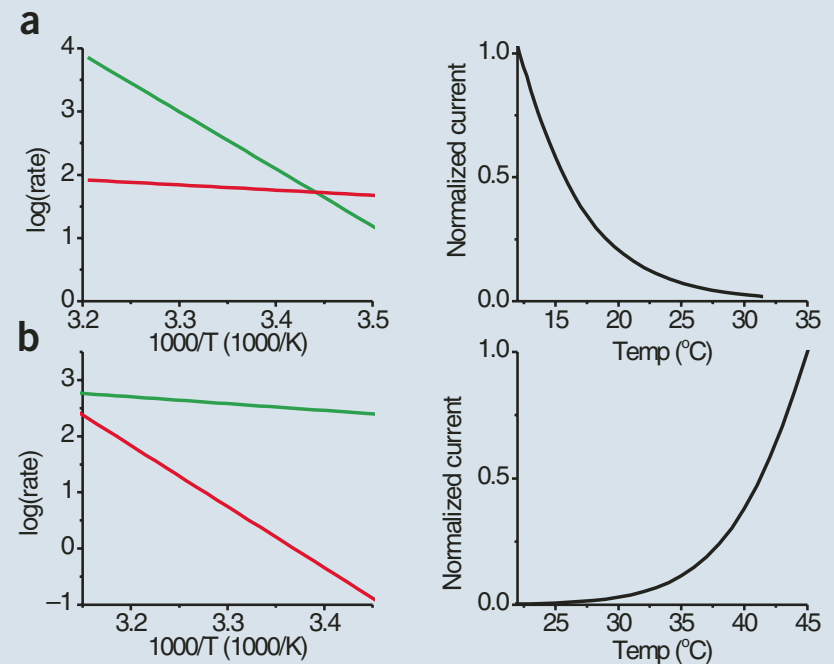

Figure 5 [AU: Please provide a title, and if desired a brief description.]

Analysis of the gating kinetics of TRPM8 (Fig. 5) revealed that the backward rate $\beta$ shows a much steeper temperature dependence than the forward rate $\alpha$, which leads to cold activation (Fig. 5a) ${ }^{41}$. The opposite is true for TRPV1 (Fig. 5b).

Half-maximal activation occurs when $\alpha=\beta$, which yields

$$
V_{1 / 2}=\frac{1}{z F}(\Delta H-T \Delta S)
$$

where $\Delta H$ and $\Delta S$ represent the difference in enthalpy and entropy between the open and closed state, respectively $\left(\Delta H=\Delta H_{\text {open }}-\Delta H_{\text {close; }}\right.$ $\Delta S=\Delta S_{\text {open }}-\Delta S_{\text {close }}$ ). This equation implies that $V_{1 / 2}$ changes by a factor of $-\Delta S / z F$ when temperature increases by $1^{\circ}$. Thus, $\Delta S$ is negative for a cold-activated channel and positive for a heat-activated channel. Note that a small gating charge ( $z$ ), as is the case for TRPM 8 and TRPV1, leads to larger temperature-dependent shifts.

heat ${ }^{45}$. Similar alterations in thermosensation have been observed in mice lacking TRPV4, a warmth-activated thermoTRP expressed in a variety of cell types, including keratinocytes and vascular endothelium ${ }^{46}$. It thus seems that cells other than sensory neurons, and keratinocytes in particular, can participate in mammalian thermosensation. The mecha- nisms whereby keratinocytes communicate with the sensory nervous system are still unknown. It has been suggested that keratinocytes might form synaptic-like contacts with sensory nerve cells in the skin, but structural or functional evidence for such a mode of communication is lacking at present. At any rate, these results urge a revision of the classical 
view that the epidermal layer of the skin serves purely as a protective barrier. Instead, the layer of keratinocytes can be seen as a large and continuous sensory organ directly involved in the assessment of ambient temperature.

The power of Drosophila genetics has allowed a reverse approach to studying the molecular mechanisms of thermosensation.[AU: $\mathrm{OK}$ as edited?] Genetic screens for mutants defective in heat response have led to the identification of the painless and pyrexia genes ${ }^{47,48}$, which encode the TRP channels most closely related to mammalian TRPA1. The painless mutants are defective in sensing noxious thermal $\left(>38^{\circ} \mathrm{C}\right)$ and mechanical stimuli ${ }^{47}$, whereas pyrexia mutants show[AU: OK again to make tense consistent within sentence?] altered thermal preferences and a reduced tolerance to heat stress ${ }^{48}$. In another recent study, an RNA interference strategy was used to determine the role of Drosophila TRPV, TRPM and TRPA channels in the thermotactic behavior of larvae. It was found that dTRPA1 is essential for the avoidance reaction to high temperatures along a thermal gradient, whereas TRPVs or TRPMs are not necessary for this reaction ${ }^{49}$.[AU: Sentence correct as edited?] Notably, dTRPA1 and pyrexia are heat-activated channels ${ }^{48,50}$, in contrast to mammalian TRPA1, which is activated by noxious cold 35,51 (Fig. 2). This opposite thermosensitivity in two ortholog channels could be highly instrumental in clarifying the molecular requirements for cold versus heat activation ${ }^{50}$.

\section{TRP channels as chemosensors}

The botanical connection. As already mentioned, the vanilloid receptor TRPV1 was identified during a cloning experiment that used capsaicin, the pungent extract of hot peppers, as an agonist $\mathrm{t}^{24}$. But capsaicin is certainly not the only botanical compound that acts directly on TRP channels. A growing number of structurally unrelated botanical compounds have been identified as potent activators of TRP channels from different subfamilies. ThermoTRPs, in particular, seem to have evolved as the favorite targets for plantderived chemicals (Table 1). The finding that a single molecule is responsible for detecting both thermal and chemical stimuli explains why we sometimes attribute inherent thermal features to food ingredients, as in 'hot' chili peppers or 'cool' mint.

Other than capsaicin, TRPV1 is also activated by resiniferatoxin, an active compound from the cactus Euphorbia resinifera that has been in medicinal use for more than 2,000 years ${ }^{52}$, and by piperine, the pungent component in black pepper ${ }^{53}$. Camphor, the waxy substance with a penetrating odor extracted from the laurel Cinnamomum camphora, acts as an agonist of TRPV3 (ref. 45). TRPM8 is directly activated by menthol and eucalyptol, two cooling compounds extracted from the mint plant Mentha piperita and the tree Eucalyptus globulus, respectively ${ }^{33,34}$. TRPA1 acts as a receptor for isothiocyanates (the pungent component in mustard, horseradish and wasabi), cinnamaldehyde (an
Table 1 Chemical agonists for thermoTRPs

\begin{tabular}{|c|c|c|c|c|}
\hline Name & Structure & Occurence & Target & Concentration \\
\hline Capsaicin & & Plant-derived & TRPV1 & $10^{-8}-10^{-6}$ \\
\hline Resiniferatoxin & & Plant-derived & TRPV1 & $10^{-10}-10^{-8}$ \\
\hline Piperine & & Plant-derived & TRPV1 & $10^{-5}-10^{-4}$ \\
\hline Olvanil & & Synthetic & TRPV1 & $10^{-8}-10^{-6}$ \\
\hline Anandamide & & Endogenous & TRPV1 & $10^{-6}-10^{-4}$ \\
\hline 2-APB & & Synthetic & $\begin{array}{l}\text { TRPV3 > } \\
\text { TRPV1 > } \\
\text { TRPV2 }\end{array}$ & $10^{-5}-10^{-3}$ \\
\hline Camphor & & Plant-derived & TRPV3 & $10^{-4}-10^{-2}$ \\
\hline $4 \alpha-P D D$ & & Synthetic & TRPV4 & $10^{-7}-10^{-5}$ \\
\hline 5',6'-EET & & Endogenous & TRPV4 & $10^{-8}-10^{-6}$ \\
\hline Menthol & & Plant-derived & TRPM8 & $10^{-5}-10^{-3}$ \\
\hline Eucalyptol & & Plant-derived & TRPM8 & $10^{-4}-10^{-2}$ \\
\hline Icilin & & Synthetic & $\begin{array}{l}\text { TRPM8 > } \\
\text { TRPA1 }\end{array}$ & $10^{-7}-10^{-4}$ \\
\hline Cinnamaldehyde & & Plant-derived & TRPA1 & $10^{-5}-10^{-4}$ \\
\hline Allylisothiocyanate & & Plant-derived & TRPA1 & $10^{-5}-10^{-4}$ \\
\hline$\Delta^{9}$-Tetrahydrocannabinol & & Plant-derived & TRPA1 & $10^{-5}-10^{-4}$ \\
\hline Allicin & & Plant-derived & $\begin{array}{l}\text { TRPA1 > } \\
\text { TRPV1 }\end{array}$ & $10^{-7}-10^{-4}$ \\
\hline
\end{tabular}

active compound in cinnamon oil) and $\Delta^{9}$-tetrahydrocannabinol (the psychoactive compound in marijuana (Cannabis sativa) $)^{36,51}$. Allicin, an unstable component of fresh garlic (Allium sativum), is an agonist for both TRPV1 and TRPA1 (ref. 54). Undoubtedly, this list of botanical TRP agonists is far from complete.

A multitude of TRP ligands. In addition to these botanical compounds, there is a long (and growing) list of synthetic TRP channel ligands. A selection of the most widely used synthetic agonists for thermoTRPs is included in Table 1. Some of these synthetic ligands show relatively high selectivity for distinct TRP channels-for example, olvanil (TRPV1) and $4 \alpha$-phorbol-12,13-didecanoate ( $4 \alpha$-PDD; TRPV4; ref. 55)[AU: Sentence $\mathrm{OK}$ as edited?] — which makes them important and widely used pharmacological tools. Others, such as 2-aminoethyl diphenyl- 
borinate (2-APB) and its analog diphenylboronic anhydride, act with comparable potency on more than one thermoTRP. The most potent activator of TRPM8 is icilin, a synthetic compound that produces intense shivering when injected into mammalian test animals ${ }^{33}$. It also activates the other cold-activated thermoTRP, TRPA1, although at 10to 100 -fold higher concentrations ${ }^{35}$. Interestingly, activation of TRPM8 by icilin (but not by cold or menthol) requires a rise in intracellular $\mathrm{Ca}^{2+}$, illustrating that this channel can act as a coincidence detector ${ }^{56}$.

In contrast to the many exogenous botanical and synthetic thermoTRP agonists, few endogenous ligands have been identified.[AU: Sentence $\mathrm{OK}$ as edited to make the two concepts being contrasted parallel?] Best described is the activation of TRPV1 and TRPV4 by several compounds related to arachidonic acid (AA; Table 1). The amide anandamide (arachidonoylethanolamide) ${ }^{57}$, as well as lipoxygenase metabolites of AA such as 12,15-(S)-hydroperoxyeicosatetraenoic acid and leukotriene $\mathrm{B}_{4}$ (ref. 58), activate TRPV1 at low micromolar concentrations. 5',6'-Epoxieicosatrienoic acid (5',6'-EET), a cytochrome P450 epoxygenase-dependent metabolite of AA, activates TRPV4 at submicromolar concentrations in a membrane-delimited manner ${ }^{59}$.

Ligand binding sites on TRP channels? At present, little is known about the interaction sites for the different TRP channel agonists on their respective targets. On the basis of the observation that the chicken ortholog of TRPV1 is insensitive to capsaicin, Jordt and Julius identified a tyrosine-serine motif (Y511 and S512) in the linker between TM2 and TM3 as an essential molecular determinant for vanilloid binding ${ }^{60}$. Additional crucial amino acid residues were identified in the $\mathrm{N}$ and $\mathrm{C}$ termini of TRPV1 (ref. 61) .The TM2-TM3 linker also contains critical determinants for the activation of TRPV4 by $4 \alpha-$ PDD (ref. 62) and of TRPM 8 by icilin ${ }^{56}$, suggesting a conserved structural and functional topology for ligand activation of these thermoTRPs.

We recently demonstrated that menthol (TRPM8) and capsaicin (TRPV1) induce channel activation by shifting the voltage dependence of activation, such that the channels open at physiologically relevant voltages ${ }^{41}$. These findings imply that these compounds should not actually be classified as agonists but rather as modulators of voltage-dependent gating [AU: Sentence OK as edited?]. Therefore, identification of the voltage sensor in TRP channels would be instrumental to understanding how these agonists interact with their channel targets.

\section{TRP channels as mechanosensors}

The first indications that TRP channels could be involved in mechanosensation came from genetic studies in the roundworm C. elegans. Worms with mutations in the osm-9 gene, which encodes a TRPV channel expressed in sensory ASH neurons, were found to have large defects in their avoidance reactions to noxious odors, high osmolality and nose touch $^{63}$. Although the mechanosensitive properties of OSM-9 have not been directly demonstrated, these findings suggested that TRP family members could act as mechanically gated channels. This was confirmed by the identification of the mammalian OSM-9 homolog TRPV4 as a cation channel gated by hypotonic cell swelling ${ }^{64-66}$. TRPV4-deficient mice show defects in pressure sensation and osmotic regulation ${ }^{67-69}$, in addition to the altered thermosensation already mentioned ${ }^{46}$. Notably, expression of mouse TRPV4 in ASH neurons from C. elegans osm 9 mutants restores the response to hypertonicity and nose touch, but not that to noxious odors, indicating that OSM-9 and TRPV4 have similar mechanosensitive properties ${ }^{70}$. Activation upon hypotonic cell swelling has also been reported for mammalian TRPV2 (ref. 71) and TRPM3 (ref. 72), as well as for Nanchung, a TRPV channel required for hearing in Drosophila ${ }^{73}$. Further research will be required to establish whether these channels have a physiological role as mechano- or osmosensors.
In a recent painstaking effort to isolate the protein that underlies the mechanosensitive cation channel (MscCa) in Xenopus laevis oocytes, Maroto and colleagues observed an abundance of TRPC1 in the protein fraction that reconstitutes $\mathrm{MscC}$ in liposomes ${ }^{74}$. Antisense inhibition of TRPC1 expression in X. laevis abolished endogenous MscCa, whereas heterologous TRPC1 expression led to a large increase in MscCa activity. They concluded that TRPC1 forms all or part of vertebrate MscCa (ref. 74), a hypothesis that strongly challenges the general view of TRPC channels as store- or PLC-dependent channels ${ }^{15}$.

Loss-of-function mutations in the nompC gene, which encodes the founding member of the TRPN subfamily, abolish mechanotransduction in Drosophila ${ }^{75}$. Similarly, knockdown of the expression of the zebrafish ortholog leads to larval deafness and imbalance ${ }^{76}$. Although these results clearly implicate TRPN channels in mechanosensation, it remains to be shown whether they act as mechanosensitive channels.

Indirect evidence also implicates PKD2, the founding member of the TRPP subfamily, in mechanosensation in the primary cilia of kidney cells ${ }^{77}$. Antibodies to PKD2 were found to inhibit the $\mathrm{Ca}^{2+}$ influx in response to physiological fluid flow.[AU: Edited as meant? It was the antibodies to PKD2 that inhibited $\mathrm{Ca}^{2+}$ influx, not blocking these

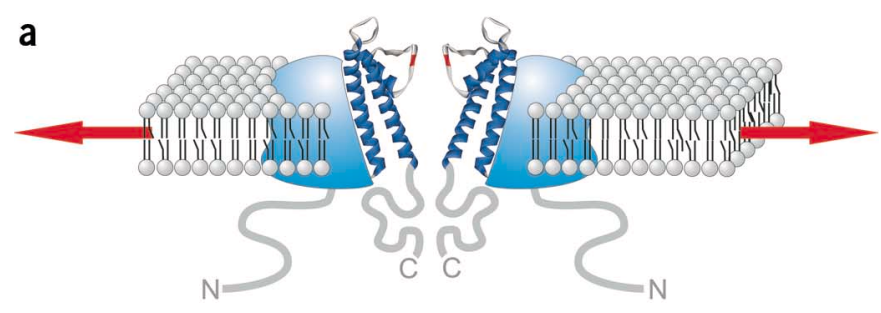

b

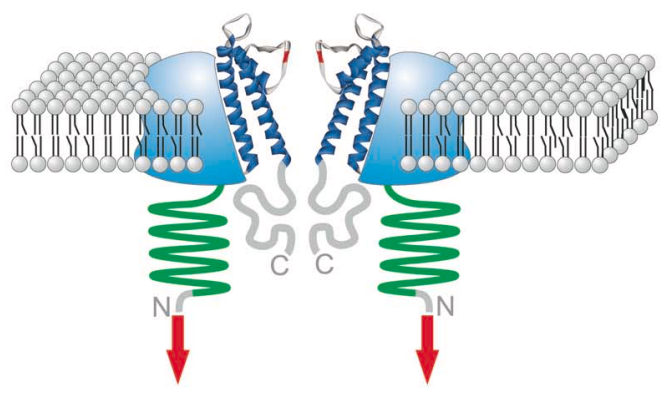

C

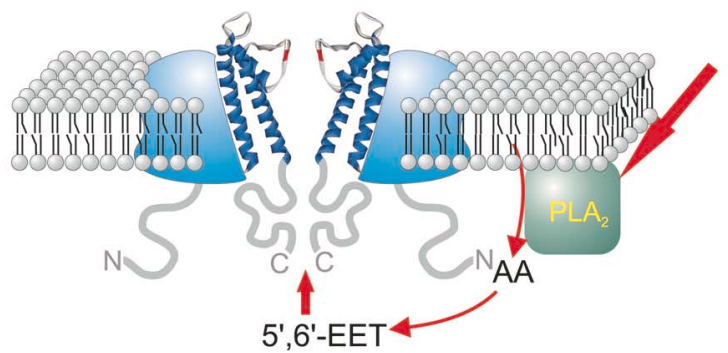

Figure 4 Converting mechanical energy into channel gating.

(a) Transmembrane domains of the channel sense the altered tension of the phospholipid bilayer induced by membrane stretch, which results in opening of the pore. (b) Mechanical stress is transduced to the channel through the cytosolic $(\mathrm{N})$ terminus, which may be attached to cytoskeletal elements and act as a gating spring. (c) Cell swelling causes activation of $\mathrm{PLA}_{2}$, leading to release of fatty acids such as AA from the membrane. Cytochrome P450 epoxygenases metabolize AA to $5^{\prime}, 6^{\prime}$-EET, which acts as a direct channel agonist for TRPV4. 
antibodies, correct?] This flow response was also abolished in cells from mice that lack PKD1, a transmembrane protein with $11 \mathrm{mem}$ brane-spanning domains that shows no homology to TRP channels. The authors of this study hypothesized that PKD1 and PKD2 assemble to form a mechanosensitive complex in the cilia of the kidney. The loss of this mechanosensitive $\mathrm{Ca}^{2+}$-influx pathway may be the basis of polycystic kidney disease. It should be noted that other studies indicate that PKD2 is expressed mainly in intracellular membranes, where it may function as a calcium-release channel ${ }^{78}$.

Another recent study implicated TRPA1 in mechanosensation, as a molecular candidate for the transduction channel in vertebrate hair cells $^{79}$. This conclusion was mainly based on three observations: TRPA1 is highly expressed in hair bundles of the hair cells, TRPA1 messenger expression coincides with the onset of hair-cell mechanosensitivity and, most importantly, disruption of TRPA1 expression in zebrafish with morpholino oligonucleotides and in mice with small-interference RNA strongly inhibited mechanotransduction in the hair cells ${ }^{79}$. It thus seems that TRPA1 is involved not only in the detection of noxious cold and pungent chemicals such as isothiocyanates and cinnamaldehyde, but also in the mechanosensitive process that forms the basis of vertebrate hearing. Clearly, a thorough study of TRPA1-deficient mice will be required to confirm this, but an educated guess is that these animals are not only less sensitive to noxious cold and mustard oil but also profoundly deaf.

Mechanisms of mechanosensation. So how do mechanosensitive TRP channels translate mechanical stimuli into channel opening? We see three possible mechanisms for the transduction of a mechanical signal to the channel gate (Fig. 4). First, mechanical stimuli may lead to changes in tension in the lipid bilayer (Fig. 4a), which can then be sensed by the transmembrane segments of the channel resulting in gating of the pore. Convincing structural evidence for such a mechanism has been presented for MscL (ref. 80), a large-conductance mechanosensitive cation channel ubiquitously expressed in bacteria. It has recently been hypothesized that TRPC1, whose mechanosensitivity is conserved after incorporation in artificial liposomes, is gated in a similar manner ${ }^{74}$. Second, mechanical stress may be transduced to the channel through its cytosolic tails (Fig. 4b), which may be connected to cytoskeletal elements. Such a mechanism has been proposed to explain the mechanosensitivity of TRPA1 and TRPN1 (refs. 79,81,82). A particular structural feature of these channels is the large number of ankyrin repeats in their $\mathrm{N}$ termini (Fig. 1b). Crystallographic studies have shown that such ankyrin repeats can form a helical structure, which has led to the hypothesis that the $\mathrm{N}$ terminus in both TRPA1 and TRPN1 acts as a gating spring ${ }^{81,82}$. Theoretical calculations of the stiffness of such an ankyrin helix yield values of around $1 \mathrm{mN} \mathrm{m}^{-1}$, which is of the same order of magnitude as the experimentally deduced stiffness of the gating spring in vertebrate hair cells ${ }^{81,82}$. Third, an enzyme whose activity induces channel gating rather than the channel itself can be mechanosensitive (Fig. $\mathbf{4 c}$ ). Evidence for such a mechanism has been presented to explain the swelling-dependent activation of TRPV4 (ref. 62). It was found that blockers of phospholipase $\mathrm{A}_{2}\left(\mathrm{PLA}_{2}\right)$ and cytochrome $\mathrm{P} 450$ epoxygenases inhibit activation of TRPV4 by hypotonic solution but not by the direct ligand $4 \alpha$-PDD. Given that cell swelling can activate PLA 2 , it was concluded that activation of TRPV4 proceeds by the $\mathrm{PLA}_{2}$-dependent formation of AA and its subsequent metabolization to 5,6'-EET (ref. 62), which then acts as a channel-activating ligand ${ }^{59}$.

\section{Concluding remarks}

The discovery of the TRP superfamily represented an important advance in our molecular understanding of sensation. Still, we are only beginning to understand how these channels work and what their exact involvement is in the different sensory modalities. Future genetic studies may also tell whether mutant TRP-channel genes lie at the basis of certain human sensory diseases, such as hearing loss or allodynia. Finally, it might be possible to couple TRP channels to silicon chips ${ }^{83}$. Such a marriage would permit use of the remarkable sensing capabilities of TRP channels for the development of new versatile biosensors.

\section{ACKNOWLEDGMENTS}

Research in the authors' laboratory is supported by the Human Frontiers Science Programme (HFSP Research Grant Ref. RGP 32/2004), the Belgian Federal Government, the Flemish Government and the Onderzoeksraad KU Leuven (GOA 2004/07, FWO G.0214.99, FWO G.0136.00, FWO G.0172.03, and the Interuniversity Poles of Attraction program, Prime Minister's Office IUAP).

\section{COMPETING INTERESTS STATEMENT}

The authors declare that they have no competing financial interests.

Published online at http://www.nature.com/naturechemicalbiology/

1. Hille, B. Ion channels of Excitable Membranes (Sinauer Associates, Sunderland, Massachusetts, 2001).

2. MacKinnon, R. Potassium channels and the atomic basis of selective ion conduction (Nobel Lecture). Angew. Chem. Int. Edn Engl. 43, 4265-4277 (2004).

3. Cosens, D.J. \& Manning, A. Abnormal electroretinogram from a Drosophila mutant Nature 224, 285-287 (1969).

4. Montell, C. \& Rubin, G.M. Molecular characterization of the Drosophila trp locus: a putative integral membrane protein required for phototransduction. Neuron 2, 13131323 (1989).

5. Hardie, R.C. \& Minke, B. The trp gene is essential for a light-activated $\mathrm{Ca}^{2+}$ channel in Drosophila photoreceptors. Neuron 8, 643-651 (1992).

6. Phillips, A.M., Bull, A. \& Kelly, L.E. Identification of a Drosophila gene encoding a calmodulin-binding protein with homology to the trp phototransduction gene. Neuron 8, 631-642 (1992)

7. Xu, X.Z., Chien, F., Butler, A., Salkoff, L. \& Montell, C. TRPgamma, a Drosophila TRPrelated subunit, forms a regulated cation channel with TRPL. Neuron 26, 647-657 (2000).

8. Reuss, H., Mojet, M.H., Chyb, S. \& Hardie, R.C. In vivo analysis of the Drosophila light-sensitive channels, TRP and TRPL. Neuron 19, 1249-1259 (1997).

9. Hardie, R.C. \& Raghu, P. Visual transduction in Drosophila. Nature 413, 186-193 (2001).

10. Chyb, S., Raghu, P. \& Hardie, R.C. Polyunsaturated fatty acids activate the Drosophila light-sensitive channels TRP and TRPL. Nature 397, 255-259 (1999).

11. Petersen, C.C., Berridge, M.J., Borgese, M.F. \& Bennett, D.L. Putative capacitative calcium entry channels: expression of Drosophila trp and evidence for the existence of vertebrate homologues. Biochem. J. 311, 41-44 (1995).

12. Wes, P.D. et al. TRPC1, a human homolog of a Drosophila store-operated channel. Proc. Natl. Acad. Sci. USA 92, 9652-9656 (1995).

13. Vriens, J., Owsianik, G., Voets, T., Droogmans, G. \& Nilius, B. Invertebrate TRP proteins as functional models for mammalian channels. Pflugers Arch. 449, 213-226 (2004).

14. Montell, C. The TRP superfamily of cation channels. Sci. STKE 2005, re3 [AU: Please confirm page number.](2005).

15. Clapham, D.E. TRP channels as cellular sensors. Nature 426, 517-524 (2003).

16. Yellen, G. The voltage-gated potassium channels and their relatives. Nature $419,35-42$ (2002).

17. Hoenderop, J.G. et al. Homo- and heterotetrameric architecture of the epithelial $\mathrm{Ca}^{2+}$ channels, TRPV5 and TRPV6. EMBO J. 22, 776-785 (2003).[AU: Please note that PubMed reported the first author as Hoenderop JG; hence this has been changed to match the PubMed reference.]

18. Voets, T., Janssens, A., Droogmans, G. \& Nilius, B. Outer pore architecture of a $\mathrm{Ca}^{2+}$ selective TRP channel. J. Biol. Chem. 279, 15223-15230 (2004).

19. Fleig, A. \& Penner, R. The TRPM ion channel subfamily: molecular, biophysical and functional features. Trends Pharmacol. Sci. 25, 633-639 (2004).

20. Birnbaumer, L. et al. On the molecular basis and regulation of cellular capacitative calcium entry: roles for Trp proteins. Proc. Natl. Acad. Sci. USA 93, 15195-15202 (1996).

21. Clapham, D.E. TRP is cracked but is CRAC TRP? Neuron 16, 1069-1072 (1996).

22. Zhu, X. et al. trp, a novel mammalian gene family essential for agonist-activated capacitative $\mathrm{Ca}^{2+}$ entry. Cell 85, 661-671 (1996).

23. Zitt, C. et al. Cloning and functional expression of a human $\mathrm{Ca}^{2+}$-permeable cation channel activated by calcium store depletion. Neuron 16, 1189-1196 (1996).

24. Caterina, M.J. et al. The capsaicin receptor: a heat-activated ion channel in the pain pathway. Nature 389, 816-824 (1997).

25. Montell, C. et al. A unified nomenclature for the superfamily of TRP cation channels. Mol. Cell 9, 229-231 (2002).

26. Patapoutian, A., Peier, A.M., Story, G.M. \& Viswanath, V. ThermoTRP channels and beyond: mechanisms of temperature sensation. Nat. Rev. Neurosci. 4, 529-539 (2003). 
27. Caterina, M.J., Rosen, T.A., Tominaga, M., Brake, A.J. \& Julius, D. A capsaicin-receptor homologue with a high threshold for noxious heat. Nature 398, 436-441 (1999).

28. Xu, $\mathrm{H}$. et al. TRPV3 is a calcium-permeable temperature-sensitive cation channel. Nature 418, 181-186 (2002).

29. Peier, A.M. et al. A heat-sensitive TRP channel expressed in keratinocytes. Science 296, 2046-2049 (2002).

30. Smith, G.D. et al. TRPV3 is a temperature-sensitive vanilloid receptor-like protein. Nature 418, 186-190 (2002).

31. Güler, A.D. et al. Heat-evoked activation of the ion channel, TRPV4. J. Neurosci. 22 , 6408-6414 (2002).

32. Watanabe, H. et al. Heat-evoked activation of TRPV4 channels in a HEK293 cell expression system and in native mouse aorta endothelial cells. J. Biol. Chem. 277, 47044-47051 (2002).

33. McKemy, D.D., Neuhäusser, W.M. \& Julius, D. Identification of a cold receptor reveals a general role for TRP channels in thermosensation. Nature 416, 52-58 (2002).

34. Peier, A.M. et al. A TRP channel that senses cold stimuli and menthol. Cell 108, 705-715 (2002)

35. Story, G.M. et al. ANKTM1, a TRP-like channel expressed in nociceptive neurons, is activated by cold temperatures. Cell 112, 819-829 (2003).

36. Jordt, S.E. et al. Mustard oils and cannabinoids excite sensory nerve fibres through the TRP channel ANKTM1. Nature 427, 260-265 (2004).

37. Prescott, E.D. \& Julius, D. A modular PIP 2 binding site as a determinant of capsaicin receptor sensitivity. Science $\mathbf{3 0 0}, \mathbf{1 2 8 4 - 1 2 8 8}$ (2003).

38. Chuang, H.H. et al. Bradykinin and nerve growth factor release the capsaicin receptor from PtdIns(4,5)P2-mediated inhibition. Nature 411, 957-962 (2001).

39. Liu, B. \& Qin, F. Functional control of cold- and menthol-sensitive TRPM8 ion channels by phosphatidylinositol 4,5-bisphosphate. J. Neurosci. 25, 1674-1681 (2005).

40. Rohacs, T., Lopes, C.M., Michailidis, I. \& Logothetis, D.E. PI(4,5)P(2) regulates the activation and desensitization of TRPM8 channels through the TRP domain. Nat. Neurosci. 8, 626-634 (2005).

41. Voets, T. et al. The principle of temperature-dependent gating in cold- and heat-sensitive TRP channels. Nature 430, 748-754 (2004).

42. Brauchi, S., Orio, P. \& Latorre, R. Clues to understanding cold sensation: thermodynamics and electrophysiological analysis of the cold receptor TRPM8. Proc. Natl. Acad. Sci. USA 101, 15494-15499 (2004).

43. Caterina, M.J. et al. Impaired nociception and pain sensation in mice lacking the capsaicin receptor. Science 288, 306-313 (2000)

44. Davis, J.B. et al. Vanilloid receptor-1 is essential for inflammatory thermal hyperalgesia. Nature 405, 183-187 (2000)

45. Moqrich, A. et al. Impaired thermosensation in mice lacking TRPV3, a heat and camphor sensor in the skin. Science 307, 1468-1472 (2005).

46. Lee, H., lida, T., Mizuno, A., Suzuki, M. \& Caterina, M.J. Altered thermal selection behavior in mice lacking transient receptor potential vanilloid 4. J. Neurosci. 25, 1304-1310 (2005)

47. Tracey, W.D., Jr, Wilson, R.I., Laurent, G. \& Benzer, S. painless, a Drosophila gene essential for nociception. Cell 113, 261-273 (2003).

48. Lee, Y. et al. Pyrexia is a new thermal transient receptor potential channel endowing tolerance to high temperatures in Drosophila melanogaster. Nat. Genet. 37, 305-310 (2005).

49. Rosenzweig, M. et al. The Drosophila ortholog of vertebrate TRPA1 regulates thermotaxis. Genes Dev. 19, 419-424 (2005).

50. Viswanath, V. et al. Opposite thermosensor in fruitfly and mouse. Nature $\mathbf{4 2 3}, \mathbf{8 2 2 - 8 2 3}$ (2003).

51. Bandell, M. et al. Noxious cold ion channel TRPA1 is activated by pungent compounds and bradykinin. Neuron 41, 849-857 (2004).

52. Szallasi, A., Blumberg, P.M., Annicelli, L.L., Krause, J.E. \& Cortright, D.N. The cloned rat vanilloid receptor VR1 mediates both R-type binding and C-type calcium response in dorsal root ganglion neurons. Mol. Pharmacol. 56, 581-587 (1999).

53. McNamara, F.N., Randall, A. \& Gunthorpe, M.J. Effects of piperine, the pungent component of black pepper, at the human vanilloid receptor (TRPV1). Br. J. Pharmacol. 144, 781-790 (2005).

54. Macpherson, L. et al. The pungency of garlic: activation of TRPA1 and TRPV1 in response to allicin. Curr. Biol. (in the press) (2005).

55. Watanabe, $\mathrm{H}$. et al. Activation of TRPV4 channels (hVRL-2/mTRP12) by phorbol derivatives. J. Biol. Chem. 277, 13569-13577 (2002).
56. Chuang, H.H., Neuhausser, W.M. \& Julius, D. The super-cooling agent icilin reveals a mechanism of coincidence detection by a temperature-sensitive TRP channel. Neuron 43, 859-869 (2004).

57. Zygmunt, P.M. et al. Vanilloid receptors on sensory nerves mediate the vasodilator action of anandamide. Nature 400, 452-457 (1999).

58. Hwang, S.W. et al. Direct activation of capsaicin receptors by products of lipoxygenases: endogenous capsaicin-like substances. Proc. Natl. Acad. Sci. USA 97, 6155-6160 (2000).

59. Watanabe, H. et al. Anandamide and arachidonic acid use epoxyeicosatrienoic acids to activate TRPV4 channels. Nature 424, 434-438 (2003).

60. Jordt, S.E. \& Julius, D. Molecular basis for species-specific sensitivity to "hot" chili peppers. Cell 108, 421-430 (2002).

61. Jung, J. et al. Agonist recognition sites in the cytosolic tails of vanilloid receptor 1 . J. Biol. Chem. 277, 44448-44454 (2002).

62. Vriens, J. et al. Cell swelling, heat, and chemical agonists use distinct pathways for the activation of the cation channel TRPV4. Proc. Natl. Acad. Sci. USA 101, 396-401 (2004).

63. Colbert, H.A., Smith, T.L. \& Bargmann, C.I. OSM-9, a novel protein with structural similarity to channels, is required for olfaction, mechanosensation, and olfactory adaptation in Caenorhabditis elegans. J. Neurosci. 17, 8259-8269 (1997).

64. Liedtke, W. et al. Vanilloid receptor-related osmotically activated channel (VR-OAC), a candidate vertebrate osmoreceptor. Cell 103, 525-535 (2000).

65. Strotmann, R., Harteneck, C., Nunnenmacher, K., Schultz, G. \& Plant, T.D. OTRPC4 a nonselective cation channel that confers sensitivity to extracellular osmolarity. Nat. Cell Biol. 2, 695-702 (2000).

66. Wissenbach, U., Bodding, M., Freichel, M. \& Flockerzi, V. Trp12, a novel Trp related protein from kidney. FEBS Lett. 485, 127-134 (2000).

67. Suzuki, M., Mizuno, A., Kodaira, K. \& Imai, M. Impaired pressure sensation in mice lacking TRPV4. J. Biol. Chem. 278, 22664-22668 (2003).

68. Mizuno, A., Matsumoto, N., Imai, M. \& Suzuki, M. Impaired osmotic sensation in mice lacking TRPV4. Am. J. Physiol. 285, C96-101 (2003).[AU: Note that PubMed could not locate this reference; please confirm accuracy.]

69. Liedtke, W. \& Friedman, J.M. Abnormal osmotic regulation in trpv4-/- mice. Proc Natl. Acad. Sci. USA 100, 13698-13703 (2003).

70. Liedtke, W., Tobin, D.M., Bargmann, C.I. \& Friedman, J.M. Mammalian TRPV4 (VR$\mathrm{OAC}$ ) directs behavioral responses to osmotic and mechanical stimuli in Caenorhabditis elegans. Proc. Natl. Acad. Sci. USA 100, 14531-14536 (2003).

71. Muraki, K. et al. TRPV2 is a component of osmotically sensitive cation channels in murine aortic myocytes. Circ. Res. 93, 829-838 (2003).

72. Grimm, C., Kraft, R., Sauerbruch, S., Schultz, G. \& Harteneck, C. Molecular and functional characterization of the melastatin-related cation channel TRPM3. J. Biol. Chem. 278, 21493-21501 (2003).

73. Kim, J. et al. A TRPV family ion channel required for hearing in Drosophila. Nature 424, 81-84 (2003).

74. Maroto, R. et al. TRPC1 forms the stretch-activated cation channel in vertebrate cells. Nat. Cell Biol. 7, 179-185 (2005).

75. Walker, R.G., Willingham, A.T. \& Zuker, C.S. A Drosophila mechanosensory transduction channel. Science 287, 2229-2234 (2000).

76. Sidi, S., Friedrich, R.W. \& Nicolson, T. NompC TRP channel required for vertebrate sensory hair cell mechanotransduction. Science 301, 96-99 (2003).

77. Nauli, S.M. et al. Polycystins 1 and 2 mediate mechanosensation in the primary cilium of kidney cells. Nat. Genet. 33, 129-137 (2003).

78. Koulen, P. et al. Polycystin-2 is an intracellular calcium release channel. Nat. Cell Biol. 4, 191-197 (2002).

79. Corey, D.P. et al. TRPA 1 is a candidate for the mechanosensitive transduction channel of vertebrate hair cells. Nature 432, 723-730 (2004).

80. Perozo, E., Cortes, D.M., Sompornpisut, P., Kloda, A. \& Martinac, B. Open channel structure of $\mathrm{MscL}$ and the gating mechanism of mechanosensitive channels. Nature 418, 942-948 (2002)

81. Sotomayor, M., Corey, D.P. \& Schulten, K. In search of the hair-cell gating spring elastic properties of ankyrin and cadherin repeats. Structure 13, 669-682 (2005).

82. Howard, J. \& Bechstedt, S. Hypothesis: a helix of ankyrin repeats of the NOMPC-TRP ion channel is the gating spring of mechanoreceptors. Curr. Biol. 14, R224-R226 (2004).

83. Neher, E. Molecular biology meets microelectronics. Nat. Biotechnol. 19, 114 (2001).[AU: Note that PubMed could not locate this reference; please confirm accuracy.] 